Document downloaded from:

http://hdl.handle.net/10251/56648

This paper must be cited as:

Escrivá Escrivá, G.; Roldán Blay, C.; Ảlvarez Bel, CM. (2014). Electrical consumption forecast using actual data of building end-use decomposition. Energy and Buildings. 82:7381. doi:10.1016/j.enbuild.2014.07.024.

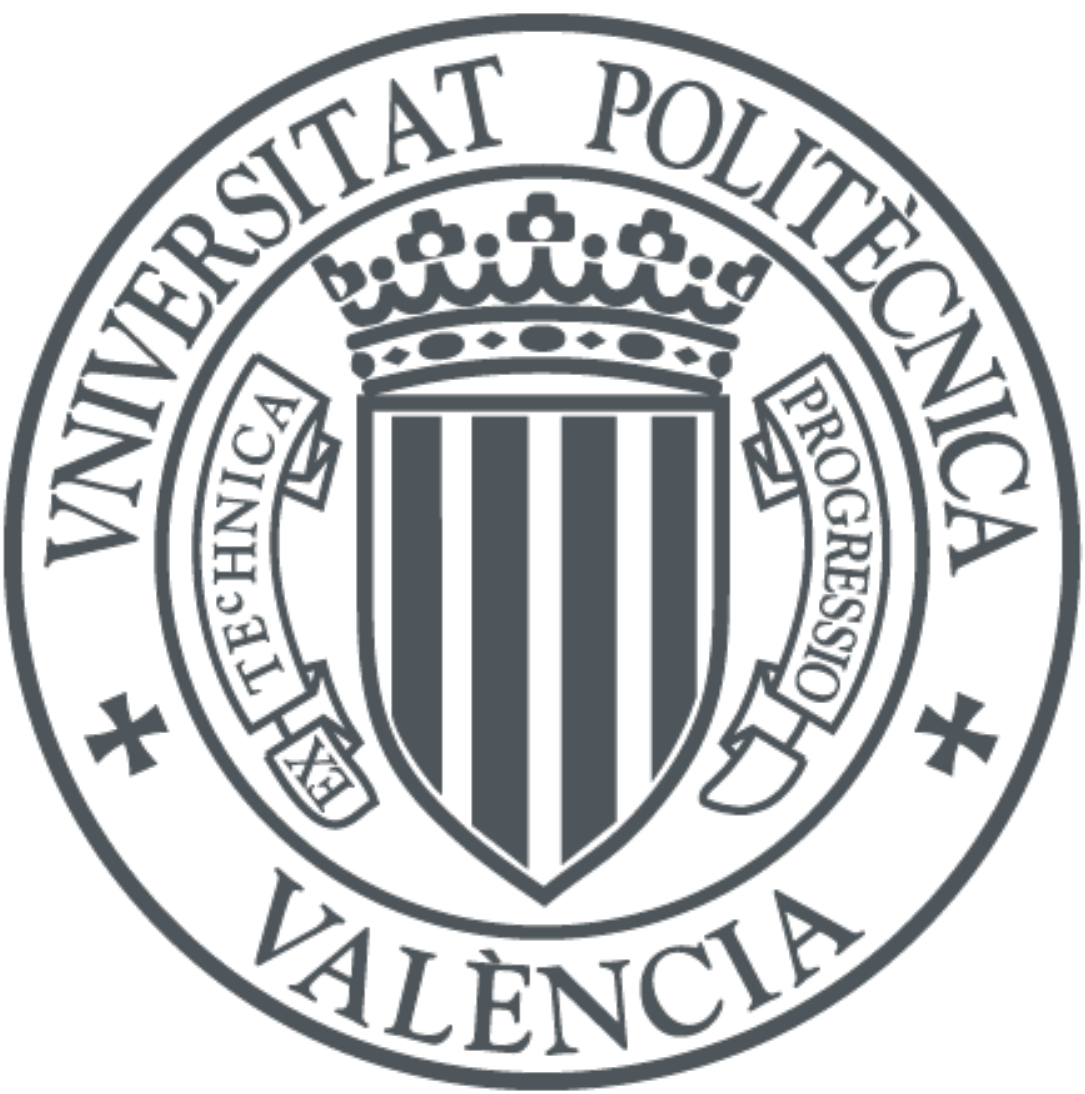

The final publication is available at

http://dx.doi.org/10.1016/j.enbuild.2014.07.024

Copyright Elsevier

Additional Information 


\title{
Electrical consumption forecast using actual data of building end- use decomposition
}

\author{
Guillermo Escrivá-Escrivá, Carlos Roldán-Blay, Carlos Álvarez-Bel \\ Institute for Energy Engineering, Universitat Politècnica de València
}

\begin{abstract}
The calculation of electricity consumption forecast a few days ahead is a complicated issue and studies about this matter are continually being performed. Advances in this field allow obtaining consumption forecasts increasingly accurate. These consumption forecasts aim to improve the knowledge of the facilities, the planning and control of consumption and the measurement and verification of energy saving measures, among others. In this study the authors present several advances related to consumption forecast using end-uses (EUs). The methodology described enables an easy disaggregation of each EU in a facility and it also enables calculating a good forecast for each of them. For the disaggregation process, the correlation between energy and external variables, such as mean temperature, degree days or daylight, is studied. Additionally, an extrapolation method to obtain a total consumption forecast from forecasted EUs that cover approximately $60 \%$ of total consumption is developed. With this procedure, total consumption forecasts with high accuracy can be obtained without the need of classifying more than the $60 \%$ of the consumption of a facility. The higher accuracy in each end-use, the better results are obtained in the total consumption forecast. For this reason, the study is focused in the end-uses disaggregation and its forecast calculation. The entire methodology is illustrated and contrasted using the consumption of the Universitat Politècnica de València.
\end{abstract}

\section{Introduction}

The energy consumption may be related to the search of different benefits. However, quantifying the benefit provided by consuming a certain amount of energy is often difficult. The best way to do this is to divide the consumption into EUs or processes to assign a benefit to each kWh of each process. This will also permit estimating the advantage of achieving a certain energy saving on an EU more accurately. But the definition of EU must allow its identification and calculation with simplicity and it must simplify, as far as possible, the forecast of that consumption to carry out the measurement and verification of energy efficiency and energy management measures. Accurate consumption forecasts are important to measurement and verification as well as to participate in the demand response programs, as it has been previously discussed in other publications of the authors [1-4]. To sum up, each consumption fraction can be dependent on external variables in a different way and these fractions are what we call EUs.

The consumption of a building or a large and complex facility can be divided, according to this criterion, into different EUs. For each EU, the forecasting process should consist of two clearly distinct steps. The first of these steps is the process of selecting the most similar days to the day of prevision (DOP). That means finding days in which external variables that affect the EU consumption have similar values to the DOP conditions. The second step is the actual forecasting process. This process can be accomplished in many different ways. In previous study made by the authors, the methodology consisted of selecting training days to use artificial neural networks to calculate the forecast. In this new study the selection process is improved and a much easier and faster extrapolation methodology that achieves very good accuracy is developed. This process calculates a forecast for each EU and the total consumption for each moment of the day using the conditions of the DOP and the conditions and consumption of selected days.

As previously discussed in other papers of the authors, the number of selected days must be reduced, near 4 days are needed [1,2]. So four days must be selected for each EU in which the variables that affect the consumer are as close as possible to the DOP.

The division of the total consumption into the different EUs provides several advantages, such as making different fractions of the consumption independent on the external variables that do not affect them. It also allows greater control and understanding of how energy is consumed. In addition, it 
simplifies the process of purchasing energy in the spot markets [5]. Furthermore, some applications oriented to specific EUs such as measurement and verification of energy efficiency and energy management actions applied on different EUs are allowed [6-8]. In conclusion, the decomposition of consumption into EUs helps the consumption forecast step in the sense that it allows the use of different input variables for the prediction of each EU, providing a series of additional benefits already discussed. A possible EUs classification is analyzed below.

The paper is organised as follows. Section 2 explains the whole methodology, introducing the necessary concepts and showing the information available related to EUs. Section 3 describes how to classify EUs. Section 4 explains how to make the consumption forecast and how to extrapolate the EUs consumption in order to calculate the total consumption forecast for the facility. Section 5 shows the results of the forecasts made to real consumption of the Universitat Politècnica de València. Finally, in Section 6 some conclusions from the study and the obtained results are drawn.

\section{Methodology}

The proposed methodology to carry out the forecast consumption of a facility consists of two stages. The first stage is the selection of days similar to the DOP. The second stage is the consumption forecast.

To carry out the days selection, a thorough analysis of the EUs of the facility is first performed. All variables that influence the consumption profile of each EU are analysed. Then, individual weights are assigned to each variable that affects the consumption of each EU. This will establish how each selection criterion affects each EU. This is necessary in order to decide which days are more similar to the DOP. The process consists of assigning qualifications to each criterion of each day depending on the similarity of each variable with the value it has for the DOP. Then, using the weights assigned to the criteria, a mark is given to each day calculated as a weighted average of the criteria qualifications. This allows ordering the days by mark to carry out the selection of four days among those which obtained the highest mark. All the days whose consumption or whose variables are considered anomalous must be removed before the days selection is made.

The criteria to be considered are external variables that affect consumption. For each EU a unique relationship between consumption and external variables can be studied. That is why the study of this relationship must be first done and different weights must be assigned to each criterion for each EU. There is a common criterion to all EUs, proximity. It has been proven over time the need to choose days surrounding the DOP to calculate an accurate consumption forecast, so this criterion must always have an important weight.

To carry out the prevision of EUs, the heuristic method described in [1] is used. Finally, an algorithm to obtain the total consumption forecast using the EUs consumption on selected days and the EUs forecast previously obtained for the DOP is applied. This algorithm performs a linear interpolation (or extrapolation if applicable) to obtain the total consumption forecast of the DOP.

The entire forecasting process is outlined in Figure 1.

The criteria that have been selected to study their relationship with the energy consumption are the following:

The average temperature (Tavg), the Cooling Degree Days (CDD), the Heating Degree Days (HDD), the maximum temperature (Tmax), minimum temperature (Tmin), the proximity to the DOP (n) and the daylight factor (DLF), a parameter that quantifies the need for artificial light of a day from its cloudiness.

The UPV has an Energy Management and Control System (EMCS) which has a total of 300 measuring points distributed along its 90 buildings. With these measuring points, consumption is measured every fifteen minutes and it is stored in a database since 2005. To classify the EUs of these facilities some of these measuring points have been classified into groups according to the type of loads connected and the behaviour in relation to external variables. Therefore, the EUs consumption curves in the UPV are the result of adding a large amount of consumption measuring points, providing a reasonably high stability to the daily consumption of each EU. 


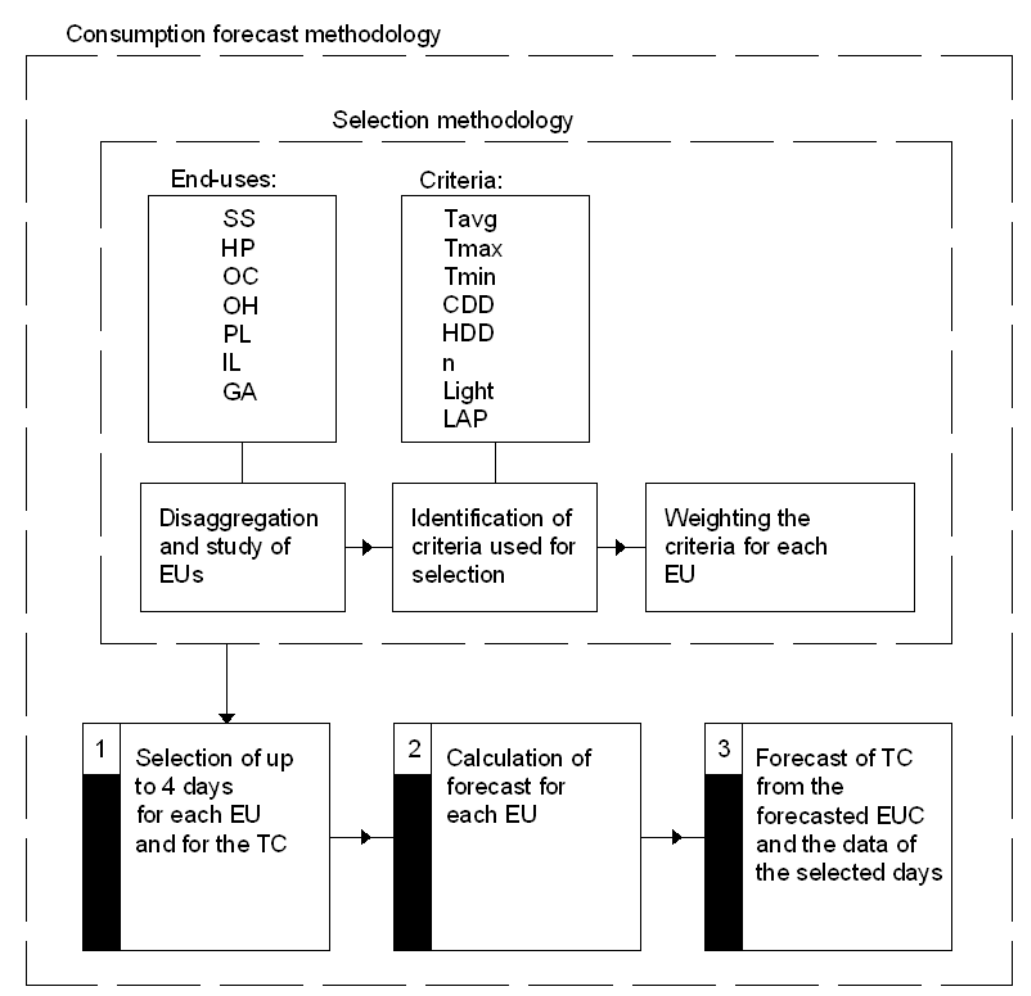

Figure 1. General diagram of the consumption forecast process.

Table 1 shows the number of measuring points that make up each EU and the main external variable that affects the consumption of each EU.

Table 1. Measuring points of EUs.

\begin{tabular}{|l|l|l|l|}
\hline EU & Description & $\begin{array}{l}\text { Number of measuring } \\
\text { points }\end{array}$ & $\begin{array}{l}\text { Main external variable that } \\
\text { depends }\end{array}$ \\
\hline SS & $\begin{array}{l}\text { Split systems } \\
\text { consumption }\end{array}$ & 25 & $\mathrm{~T}^{\underline{a}}$ \\
\hline HP & Heat pumps consumption & 36 & $\mathrm{~T}^{\mathbf{a}}$ \\
\hline OC & Only cold production & 2 & $\mathrm{~T}^{\underline{a}}$ \\
\hline OH & Only heat production & 4 & $\mathrm{~T}^{\mathrm{a}}$ \\
\hline PL & Public lighting & 15 & Day number \\
\hline IL & Internal lighting & 18 & External light \\
\hline GA & General appliances & 16 & Occupancy \\
\hline TC & Total consumption & 1 & All \\
\hline
\end{tabular}

The consumption formed by the addition of the identified EUs is what is called the end-uses consumption (EUC), which represents around $60 \%$ of the UPV's total consumption (TC). This percentage is being constantly revised and improved by identifying and classifying the type of consumption measured by new measurement points installed in the UPV. An accurate prediction of the EUC results in a good prediction of the TC simply by using a fast and easy extrapolation method explained below. Therefore, it is important to improve the selection and classification of EUs so as to get a reliable and strict relationship between consumption and external variables. This will permit finding a good days selection, similar to the DOP, in both the input variables and consumption for each EU, improving the forecast accuracy. 


\section{EUs}

First, the temperature-dependent consumption can be classified into four groups. On the one hand, the temperature-dependent consumption that has a unique behaviour for all seasons, Split Systems (SS). This is because this EU includes machines whose operation mode is automatically selected depending on the desired temperature and the external temperature. Secondly, consumptions with seasonal behaviour, such as heat pumps (HP), which operate differently depending on whether they are in heating or cooling mode and that mode switching occurs manually at a certain moment of the year. Furthermore, there is an important consumption, different from the previous ones, due to those machines that produce cold at any time of year, the only chillers systems (OC). Thus, this is a refrigeration consumption, which is only affected by high temperatures, but for low values this consumption remains at a small value (residual consumption). This consumption is formed by all the equipment refrigeration machines. Finally, the last temperature-dependent consumption is the consumption of radiators, heaters, etc., that is the only heat systems $(\mathrm{OH})$. This consumption is always producing heat, so it is only affected by temperatures lower than a certain value, and above this thermal limit the consumption of this EU is a low consumption.

Besides temperature-dependent EUs, there are other EUs, such us lighting consumption that can be separated in public lighting (PL), which is dependent on the length of the daylight hours and the interior lighting (IL), which depends on the cloudiness and the time of the day.

Finally, another EU of force can be identified, which mainly depends on the facilities occupancy, the general appliances (GA).

The behaviour of each EU in relation to a significant variable which affects its consumption is analysed below to explain the way to leverage the separation of the consumption of each EU. A relationship between daily energy of each $\mathrm{EU}$ and the selected external variable has been obtained for all the weekdays (Tuesday, Wednesday and Thursday) of 2010 for those EUs that have a dependency on the working patterns. This relationship has been studied in detail to produce the table of weights of the criteria used for selecting the days similar to the DOP. Every day whose behaviour is anomalous for any reason (measurement errors, specific events, reduced work time, etc.) has been removed in this study. The figures below show the correlation between the daily energy and a significant variable that is related to the consumption of each EU. The energy has been normalized to the energy value of the $25 \%$ percentile.

\subsection{SS}

Figure 2 shows the relationship between the daily energy of the MP EU and the average temperature of the day, for weekdays (Tuesday, Wednesday and Thursday).

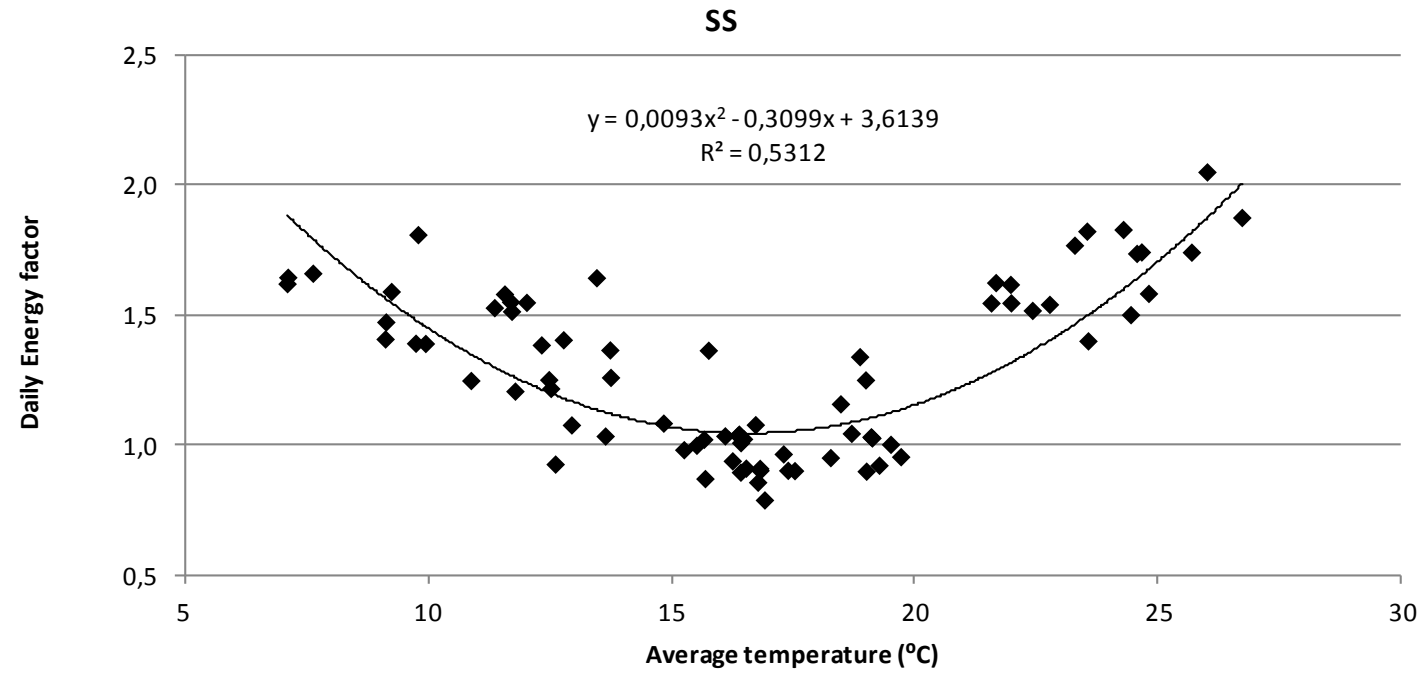

Figure 2. Relationship between the daily energy and the average temperature for weekdays for the MP EU. 
It can be seen that at low and high temperatures there is a higher consumption, with a saturation value determined by the power of the equipment, its performance and the acclimatization conditions of the facility. In this case, the average temperature has a great importance, as well as the CDD (or the HDD according to the trend of the DOP) to establish a correlation with the consumption. Furthermore, with deeper studies it is observed that the relationship between the consumption and the maximum temperature is better than the correlation between the consumption and the minimum temperature throughout the year. All this is reflected in the weights assigned to each criterion for this EU.

\section{$3.2 \mathrm{HP}$}

The following EU, HP, composed of heat pumps and air conditioning machines with manual mode switch is studied below. Figure 3 shows the correlation between the EU and the average temperature of the day.

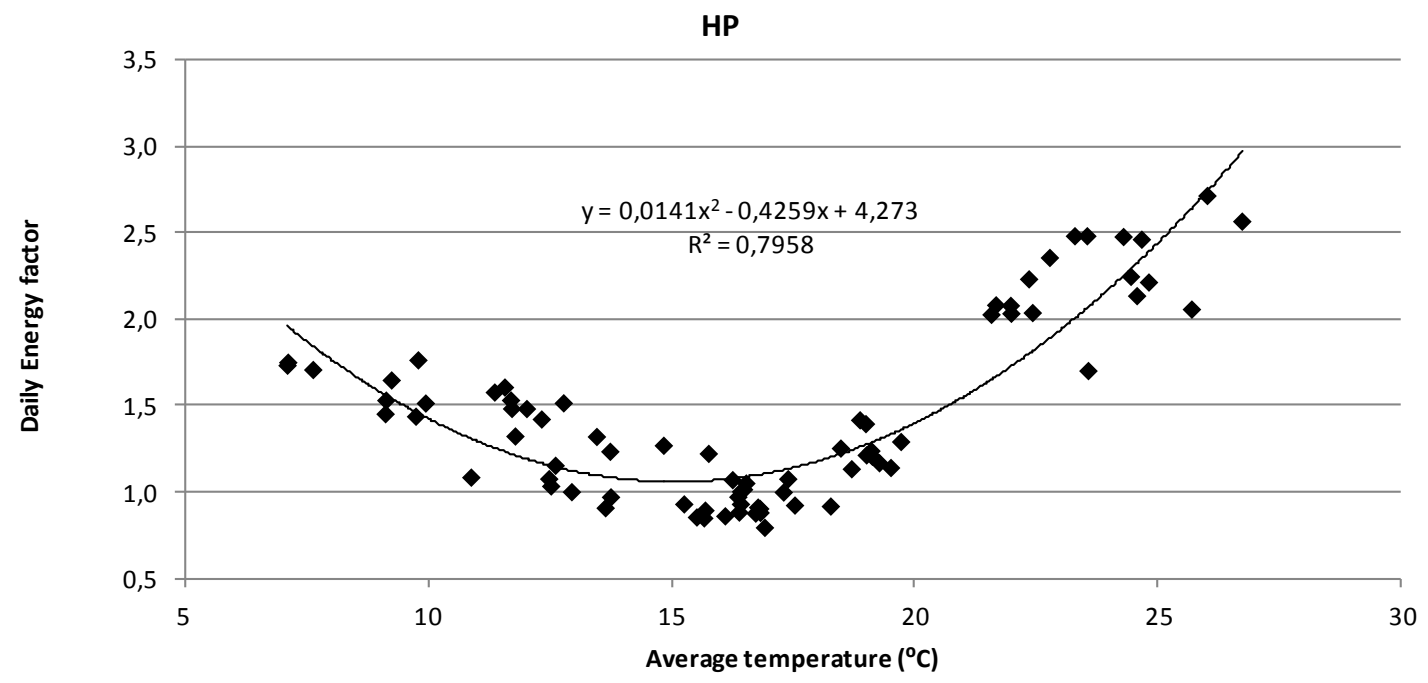

Figure 3. Correlation between the daily energy consumption and the average temperature for the HP EU.

In this EU there is a fairly good correlation, which is an important advantage of the disaggregation, since some consumption fractions are identified with a great accuracy correlation with certain input variables. However, due to the manual operation mode switch, there are two different correlations that can be separated to improve the forecast results. These correlations must be done by separating the days in which the machines operate in heating mode and the days in which they operate in cooling mode. This permits having two curves, one showing a tendency to increase at low temperatures and another one that increases at high temperatures. Figure 4 shows these two correlations.

As clearly seen, the behaviour of these machines in cooling mode is much more related to the average temperature of the day than the opposite behaviour. Therefore, due to the difference between these correlations, using days with the same operating mode than the DOP is always needed to calculate an accurate consumption forecast for this EU. Indeed, the behaviour of this EU is similar to previous one, thus, the weights are also similar, although there is slight increase in the weight of the average temperature while the maximum and minimum temperature weights are reduced. 

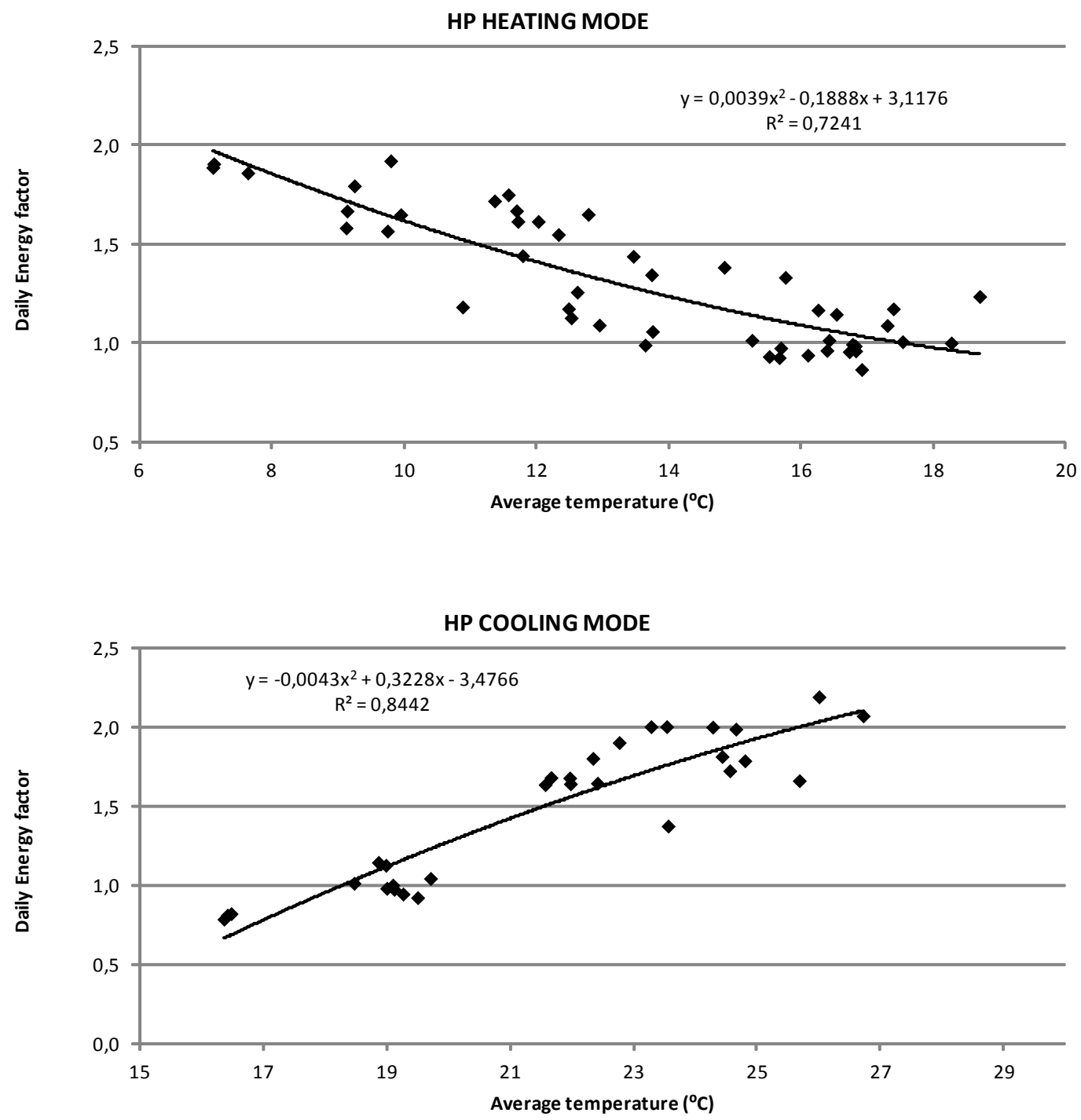

Figure 4. Correlation between the daily energy consumption and the average temperature for HP EU in both operation modes.

\section{$3.3 \mathrm{OC}$}

The two following EUs have similar behaviours to the previous figures that showed HP EU in each operating mode. This is because its consumption is used to produce only heat or only cool. Figure 5 belongs to the OC EU.

There is a good correlation between the average temperature of the day and the consumption of this EU. It can be noted that below a certain temperature the consumption remains practically constant. The relevant variables are the maximum, minimum and average temperature, as well as the CDD. 


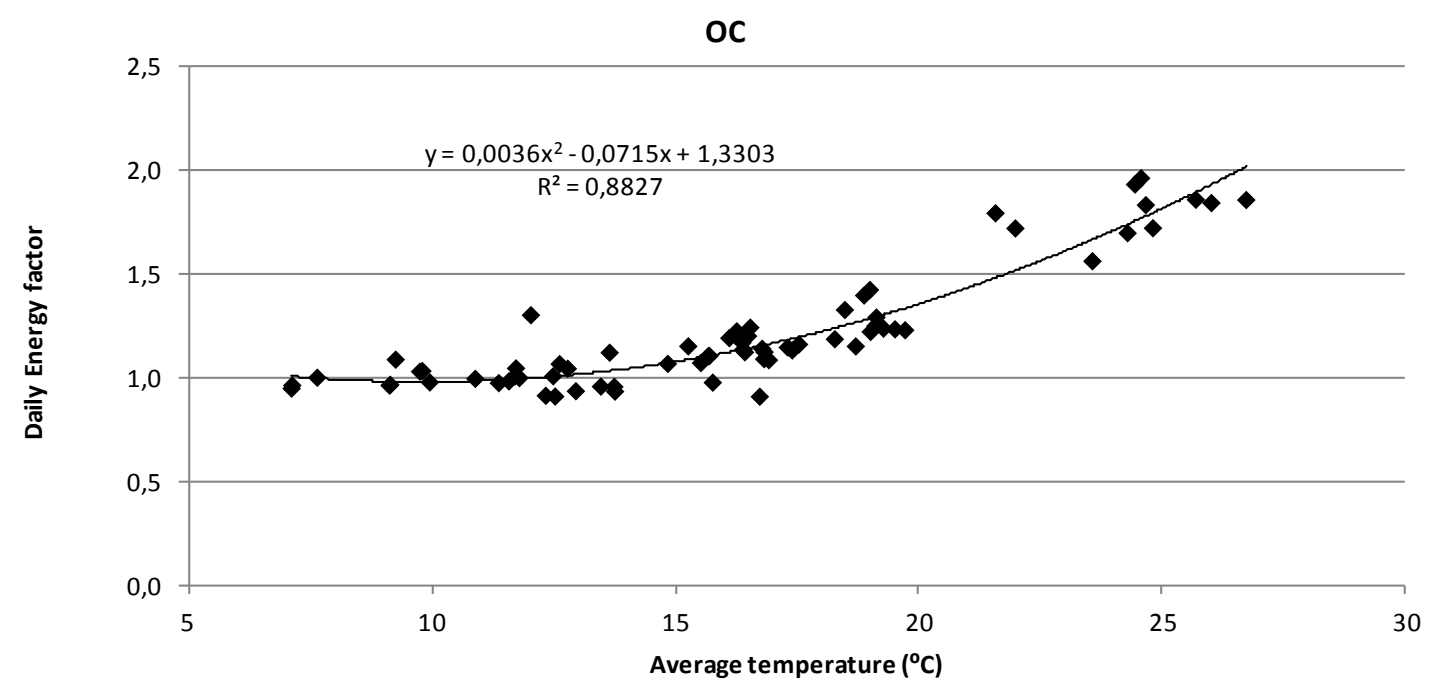

Figure 5. Correlation between the daily energy consumption and the average temperature for OC EU.

\section{$3.4 \mathrm{OH}$}

The OH EU presents the behaviour shown in Figure 6.

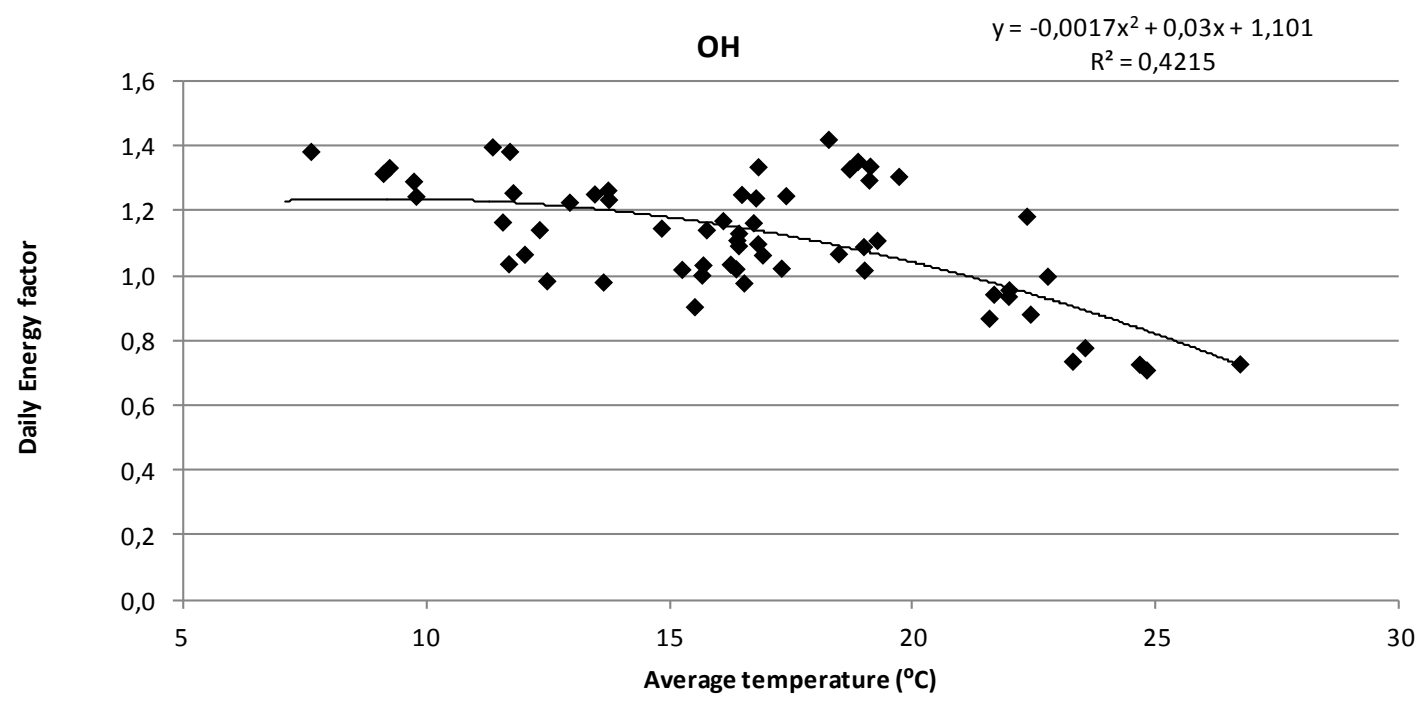

Figure 6. Correlation between the daily energy consumption and the average temperature for OH EU.

It can be seen that the average temperature is much more related to the consumption of cold production machines than with the heat production consumption. The $\mathrm{OH}$ EU presents a rather erratic behaviour, which leads to the need to ensure the use of days close to the DOP to minimize the error in the forecasts by taking close and presumably similar consumptions. The weight assigned to the thermal variables is considerably reduced as a result of the study carried out for this EU. Among these variables, the most interesting one is the HDD that indicates with better accuracy how cold a day has been. 


\subsection{PL}

The other EUs that are not temperature-dependent can be correlated with the day number of the year, since this consumption usually has a smooth evolution along the time. Thus, the resemblance between the consumption of a certain day and those around it can be noted. In the case of public lighting, the consumption is not dependent on the working patterns. The only thing that affects this consumption is the duration of the night (or equivalently the daylight length), since this lighting turns on automatically when daylight falls below 100 lux as PLCs used to control lighting lines have been programmed. The following graph shows a whole year for this EU.

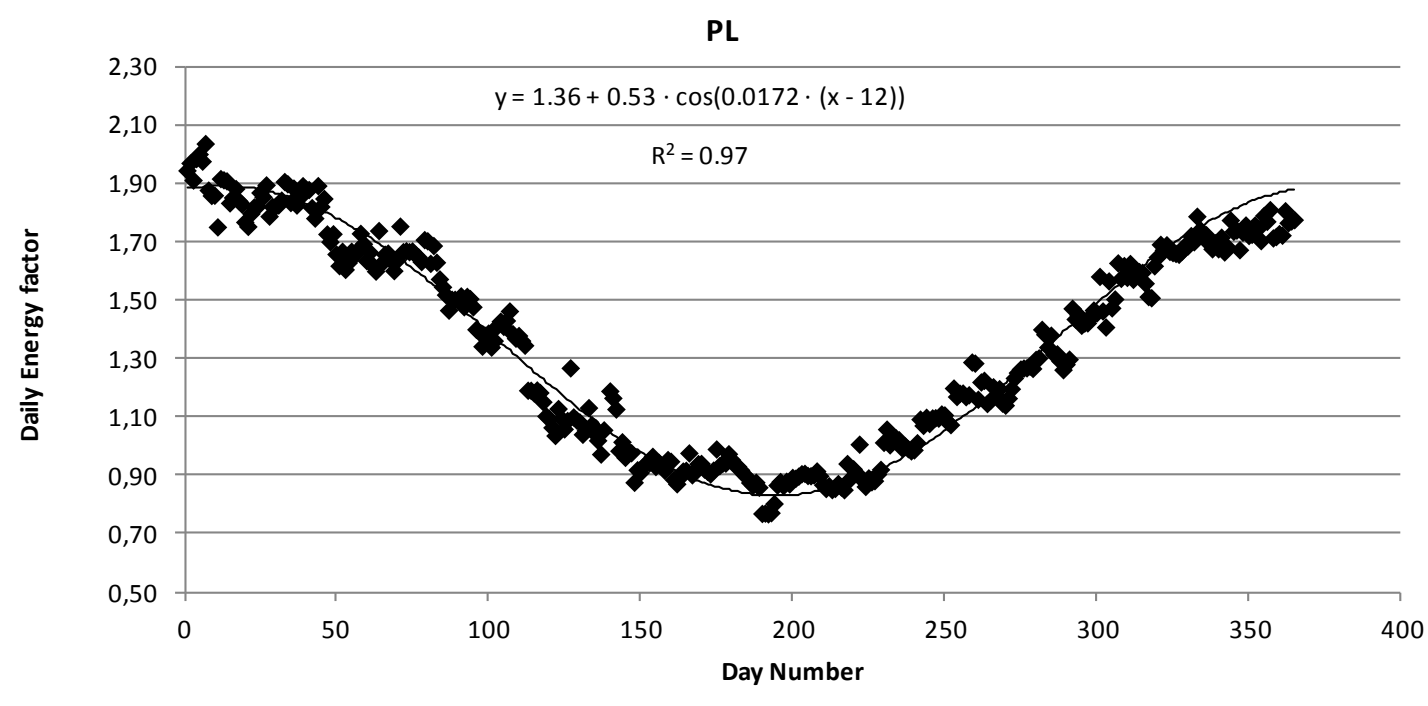

Figure 7. Consumption evolution for PL EU.

The evolution of this EU is sinusoidal, like the evolution of the daylight hours throughout the year. Therefore, in this EU, the only criterion that is taken into account is the day of the year, since the consumption is very similar for consecutive days.

\subsection{IL}

The internal lighting is similar to public lighting, but it has two important differences: it is affected by the working patterns and the consumption is high when a day is very cloudy, but is fairly constant on clear days. Figure 8 shows the behaviour of this EU.

The days show an evolution with a slight sinusoidal tendency in which each day consumes similarly to those around it. In this graph, the dependence of the consumption of this EU on natural light can be seen, since the four days with the higher cloudiness have been marked and they have interpolated with a sine curve higher than curve used to interpolate the other days of the year.

It is therefore important to choose a cloudy day if the DOP is cloudy. Otherwise, it will be needed to use the days closest to the DOP with the same LAP.

To take into account the cloudiness of a day, the daylight factor (DLF) is defined. This factor is a value calculated from the formula presented below. The DLF represents the need of artificial light depending on the measurement of radiation throughout the day. Therefore, the cloudiness of a day is defined as a value from 0 (clear) to 4,000 (totally cloudy). This value is 4000 minus the average of all values below 4.000 lux during the day from 9 am to $5 \mathrm{pm}$ :

$$
\mathrm{DLF}=\left\{\begin{array}{cl}
4,000-\left(\operatorname{avg}\left(\operatorname{lux}_{\mathrm{i}}\right)_{\forall \operatorname{lux}_{\mathrm{i}}<4,000}\right) & , \exists \mathrm{i}: \operatorname{lux}_{\mathrm{i}}<4,000 \\
0 & , \operatorname{lux}_{\mathrm{i}} \geq 4,000 \forall \mathrm{i}
\end{array}\right.
$$


IL

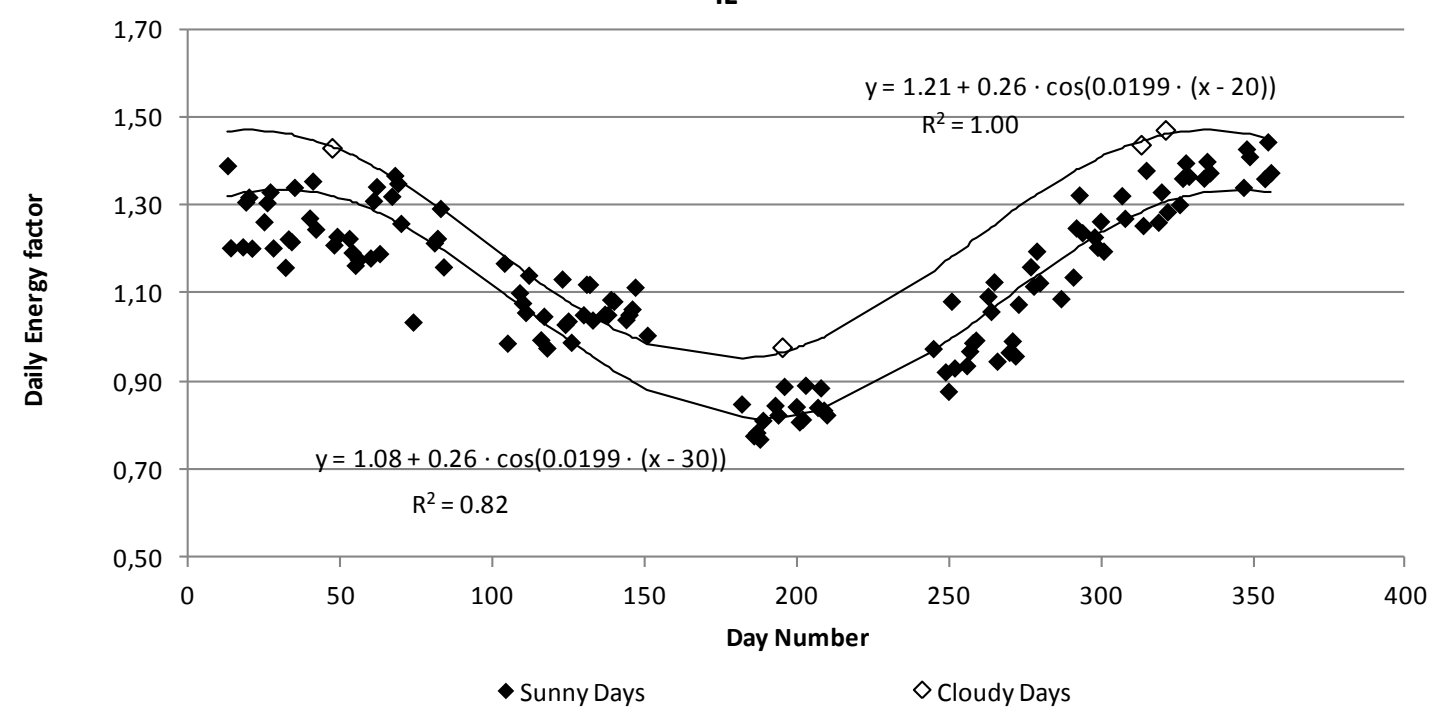

Figure 8. Consumption evolution for IL EU in cloudy and sunny days.

Therefore, lux values must be recorded every 5-15 minutes in order to obtain a good accuracy in the calculation of the DLF.

\subsection{GA}

Finally, the consumption of the general appliances depends on the occupancy of the facilities. In this consumption that exist only when staff is present on site, the most important thing is to select a day with the same LAP and as close as possible to the DOP. In Figure 9 this EU is analysed.

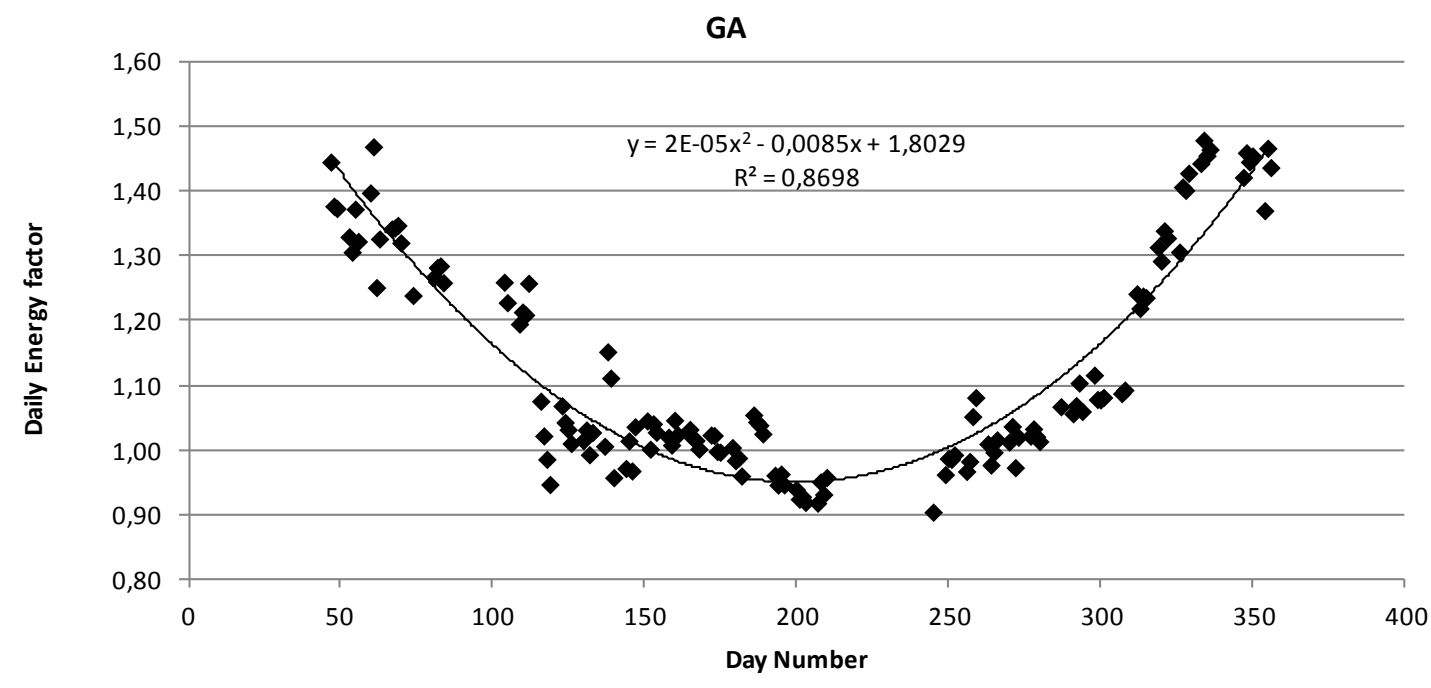

Figure 9. Consumption evolution for GA EU.

As can be seen, the correlation shown in the previous figure is very good, although in this EU large loads can be disconnected in a certain day or vice versa and so, there may be isolated consumption points with anomalous behaviours. The best option to prevent this variability from producing a significant influence in the forecast is not to use these anomalous days for future forecasts after identifying that the consumption of these days is unjustified. Moreover it is appreciated that the days surrounding a certain area usually have a similar consumption. Therefore, four days will be selected from the available days closest to the DOP to calculate a rigorous forecast of this EU. 


\section{Consumption forecast}

The criteria used to carry out the days selection has a different influence on the consumption of each EU. Due to this fact, different weights are assigned to each criterion for each EU in order to qualify dates for historical data when carrying out the selection of the most appropriate training days. This is the result of the previous study of EUs.

In addition to the criteria, the LAP can influence the consumption of a day. To take into account for this criterion this parameter is defined for each day. A labour affinity table is created to assign a labour affinity parameter to each pair of LAP values. This labour affinity parameter is a value between 0 and 1 to indicate the affinity between these two values of LAP. Thus, an affinity value of 0 implies that these are very different LAP and an affinity value of 1 means that both LAP values are considered almost identical. Intermediate values quantify the similarity between the two LAP values. The mark obtained by each day is multiplied by the affinity between the LAP of that day and the LAP of the DOP. This step can penalize or even cancel the mark of days with a working pattern different to DOP. This takes place whenever the end use consumption is affected by LAP, thereby correcting the notes assigned to each day. As a result of this step, only days with the same working patterns as the DOP will be selected. If there are no days with the same LAP, then the days with the most similar LAP values will be selected.

All the information related to the weights of the criteria, quantified according to this study is shown in Table 2.

Note that when the weights assigned to CDD and HDD are not null for an EU these values are right assuming that in the DOP CDD>HDD (it is a hot day). Otherwise, the weights should be exchanged, and if the values of CDD and HDD were very similar (e.g. $\mid$ CDD-HDD $\mid<10$ ), then each weight should be the average of both values (the weights would be divided into two equal parts).

Thus, in each end use, the selection of the four days more similar to the DOP is carried out by assigning a mark to each day and choosing the top four. These marks are calculated as the weighted average of the notes on each of the criteria. That is, for each end use, the obtained mark is:

$$
M_{i}=\left(\sum_{j=1}^{N_{\text {criveria }}} M_{i, j} \cdot W_{j}\right) \cdot L a b_{\text {factor }}
$$

Where i represents each of the possible days to be selected, $j$ is each of the criteria; Ncriteria is the total number of criteria ( 7 in this study); $\mathrm{Mi}, \mathrm{j}$ is the mark from 0 to 1 obtained by the day $\mathrm{i}$ in the criterion $\mathrm{j}$, it can be 1 if the day is identical to the DOP for this criterion or 0 if this criterion is the least similar among all the possible days, and it may be any intermediate decimal value; $\mathrm{Wj}$ is the weight of the criterion $\mathrm{j}$ for the current EU, that is shown in Table 2, and $\mathrm{Lab}_{\text {factor }}$ is a factor with a value of 1 if the LAP for the day $i$ is the same as for the DOP or if the LAP does not affect the consumption of the current EU, and a value between 0 and 1 in other cases, the value of the labour affinity parameter is used in these cases (a null affinity cancels the mark of this day, avoiding the selection of days with working patterns not related to the DOP).

The extrapolation method that has been developed and that is described below is based on linear interpolation and extrapolation with constant limits. To carry out the calculation of TC for a specific instant the EUC at that moment for the four selected days and the expected EUC for the DOP at that moment are used. Among the four chosen values only the two nearest to the expected value for the DOP are used, one above and one below. If it is not possible to select a value above and one below, the closest values will be used.

With these two values of EUC and the expected TC values for that moment, a line is drawn to do the extrapolation (if needed) allowing 15\% above and below the value of maximum and minimum TC. Once these limits are reached, two horizontal lines are used to continue the extrapolation function. This dashed function will result in the value of the forecasted TC for the DOP at the given time from the forecast of EUC for the same moment.

Figure 10 shows the extrapolation process described above. 
As shown, this is a simplification of a sigmoid function. This function provides linear values in the intermediate zone and it avoids excessively high extrapolations (limited to $15 \%$ of the total variation between both selected days).

Table 2. Weights for each EU and TC.

\begin{tabular}{|l|l|l|l|l|l|l|l|l|}
\hline & $\mathbf{T}_{\text {avg }}\left({ }^{\circ} \mathbf{C}\right)$ & $\mathbf{C D D}$ & $\mathbf{H D D}$ & $\mathbf{n}$ (day number) & $\mathbf{D L F}$ & $\mathbf{T}_{\max }\left({ }^{\circ} \mathbf{C}\right)$ & $\mathbf{T}_{\min }\left({ }^{\circ} \mathbf{C}\right)$ & LAP \\
\hline SS & 1.95 & 1.30 & 0.26 & 3.90 & 0.00 & 1.95 & 0.65 & Yes \\
\hline HP & 2.63 & 1.89 & 0.21 & 3.16 & 0.00 & 1.58 & 0.53 & Yes \\
\hline OC & 1.50 & 0.50 & 0.00 & 6.00 & 0.00 & 1.00 & 1.00 & Yes \\
\hline OH & 0.52 & 0.00 & 0.61 & 8.70 & 0.00 & 0.13 & 0.04 & Yes \\
\hline PL & 0.00 & 0.00 & 0.00 & 10.00 & 0.00 & 0.00 & 0.00 & No \\
\hline IL & 0.00 & 0.00 & 0.00 & 0.91 & 9.09 & 0.00 & 0.00 & Yes \\
\hline GA & 0.00 & 0.00 & 0.00 & 10.00 & 0.00 & 0.00 & 0.00 & Yes \\
\hline TC & 2.16 & 0.43 & 0.07 & 4.32 & 0.14 & 2.16 & 0.72 & Yes \\
\hline
\end{tabular}

\section{Application and results}

The composition of EU in the Universitat Politècnica de València is shown below. It can be seen that the greater part of them is represented by thermal EUs.

The forecast for each EU is obtained using the described methodology with the appropriate weights of the criteria to select days and applying the heuristic method to perform the calculation of the forecast, commented in section 2.

The sum of all EUs curves results in the EUC curve. This EUC forecasted curve is extrapolated to obtain the TC forecasted curve for the facility.

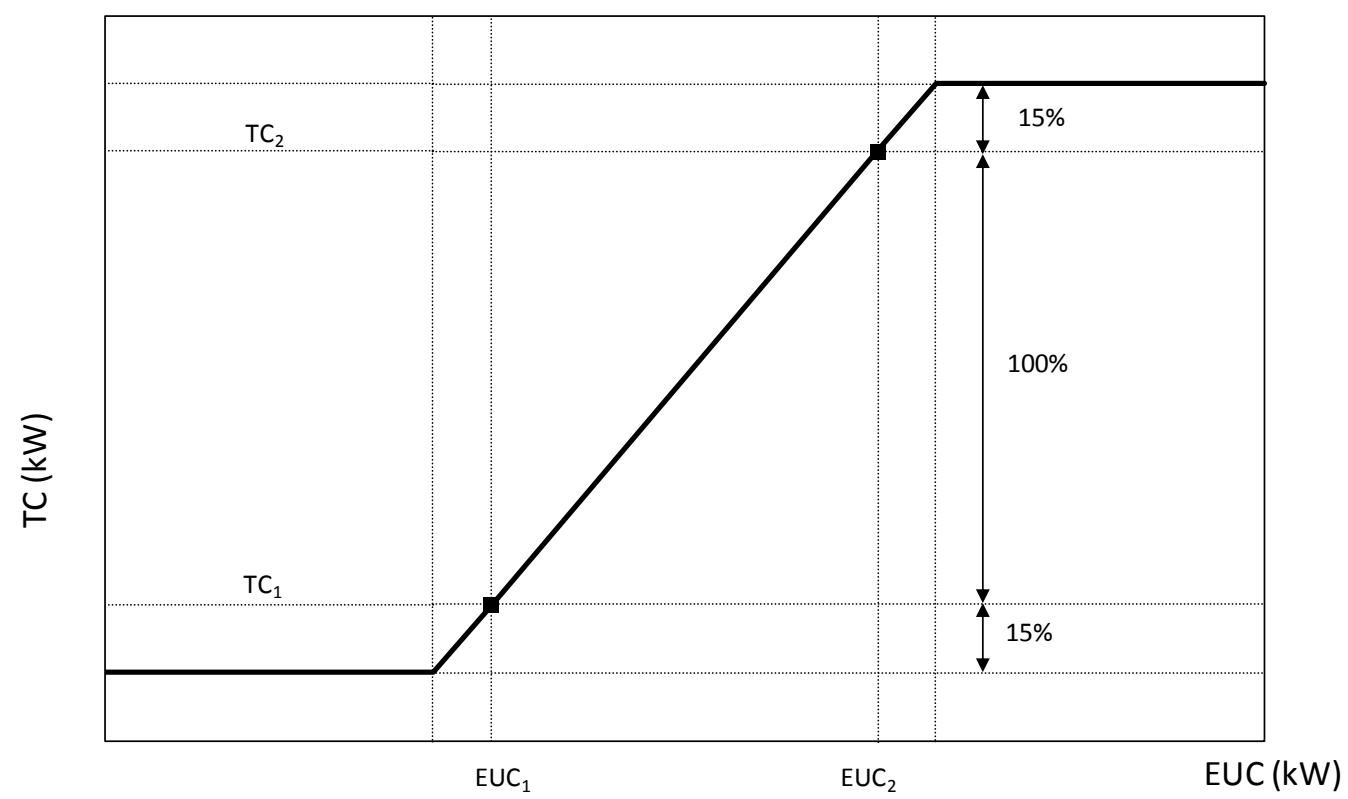

Figure 10. Function used to calculate the extrapolation of the TC from the forecasted EUC for each moment of the DOP.

Figure 12 shows the forecasted consumption of a full week for TC and EUC in the UPV. It can be seen how the errors obtained in the EUC forecast are translated into similar errors in the TC curve. From another point of view, the accuracy achieved in the calculation of the EUC forecast remains at the same level in the TC forecast. 
In the previous figure, the curves "Actual EUC" and "Actual TC" are the actual consumption curves for EUs and TC of the facility and the curves called "Forecasted EUC" and "Forecasted TC" are the forecasted curves for EUC and TC respectively.

The results for this week have an average MAPE of $3.39 \%$. The individual MAPE for each day are shown in Table 3.

Table 3. MAPE values obtained in the forecasts made for the week from 2012/3/26 to 2012/4/1.

\begin{tabular}{|l|l|l|l|l|l|l|l|l|}
\hline Date & $\mathbf{3 / 2 6 / 2 0 1 2}$ & $\mathbf{3 / 2 7 / 2 0 1 2}$ & $\mathbf{3 / 2 8 / 2 0 1 2}$ & $\mathbf{3 / 2 9 / 2 0 1 2}$ & $\mathbf{3 / 3 0 / 2 0 1 2}$ & $\mathbf{3 / 3 1 / 2 0 1 2}$ & $\mathbf{4 / 1 / 2 0 1 2}$ & Avg \\
\hline MAPE & $3.38 \%$ & $1.97 \%$ & $1.68 \%$ & $5.32 \%$ & $2.33 \%$ & $4.63 \%$ & $4.44 \%$ & $3.39 \%$ \\
\hline
\end{tabular}
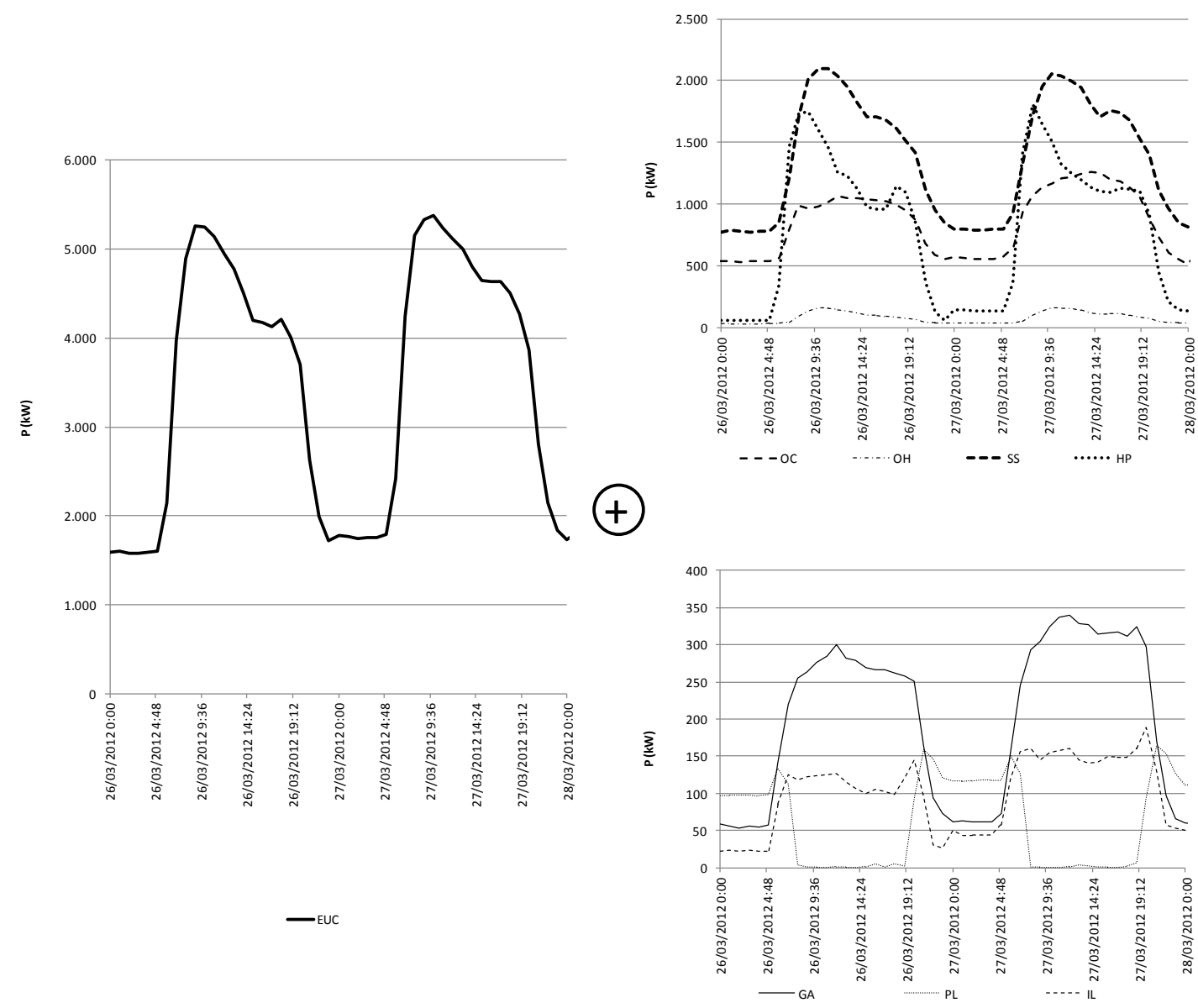

Figure 11. Composition of the addition of EUs from thermal and non-thermal EUs.

Over a whole year (from July 2011 to June 2012), the results obtained with this methodology have an average MAPE of $5.15 \%$. 


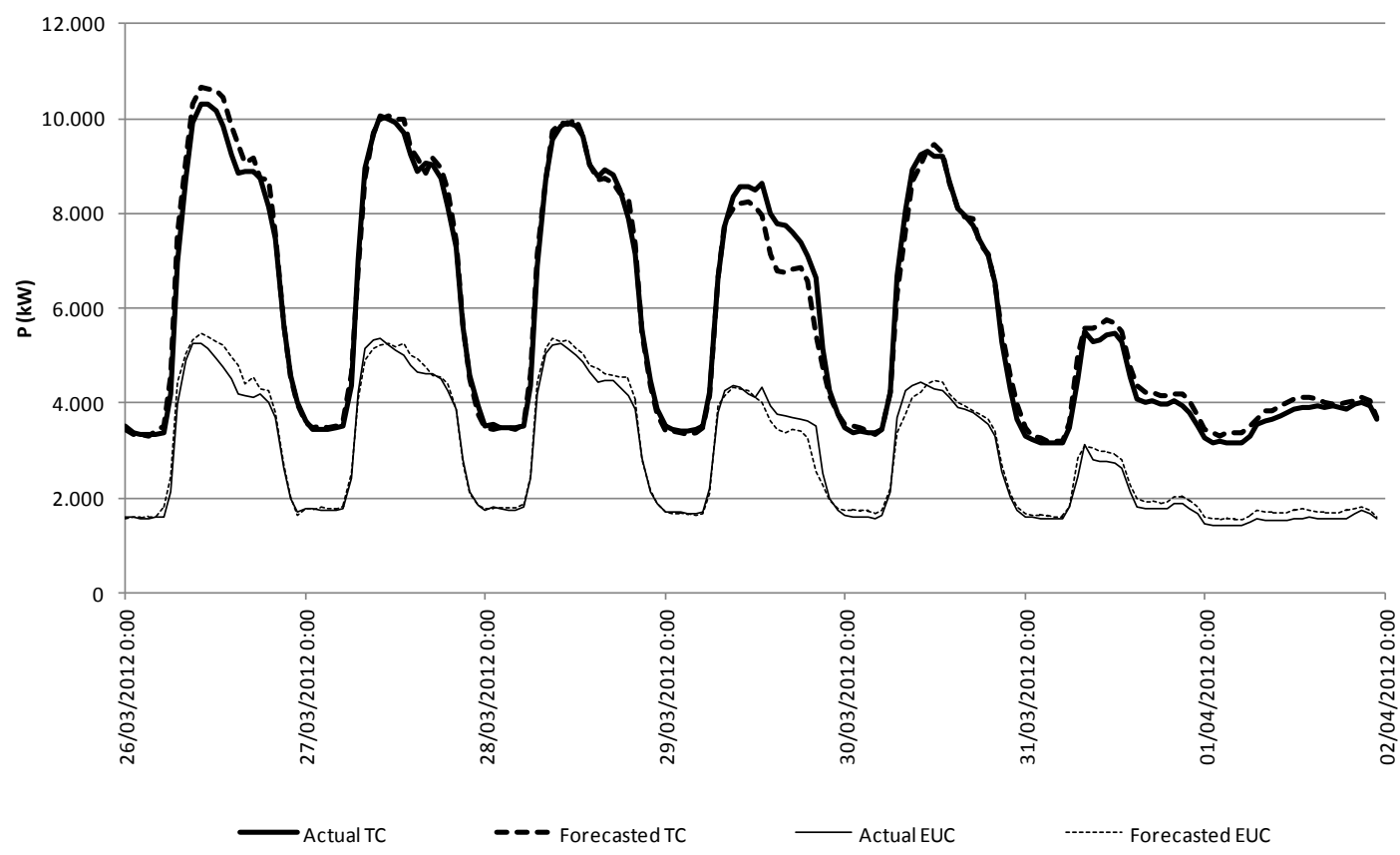

Figure 12. TC and EUC forecasts for a whole week.

\section{Conclusions}

A classifying methodology has been developed in order to disaggregate the EUs of a facility. A forecast methodology to calculate a forecast for EUC and TC has been developed and proved. The described methodology has a high accuracy in the TC forecast and it allows the calculation of the TC from a previously calculated forecast of the EUC that represents about $60 \%$ of the TC.

Each EU consists of the sum of many measuring points with similar consumption behaviour and similar energy use. This facilitates the EUs classification task and it gives them a high stability in their behaviour, which allows obtaining accurate forecasts for each of them, using the methodology described above.

The final step of the forecasting process permits the extrapolation of the EUC to the TC while maintaining a similar precision, therefore, a good precision of the forecast of about $60 \%$ of the TC will result in an accurate forecast of the TC. Thus, future work to improve these forecasts should focus on partial forecasts of each EU, in order to get highly accurate forecasts of the actual consumption.

\section{References}

[1] G. Escrivá-Escrivá, C. Álvarez-Bel, C. Roldán-Blay, M. Alcázar-Ortega, New artificial neural network prediction method based on buildings' end-uses for energy consumption forecast. Energy and Buildings 43 (2011) 3106-3111.

[2] Carlos Roldán-Blay; Guillermo Escrivá-Escrivá; Carlos Álvarez-Bel; Carlos Roldán-Porta; Javier Rodríguez-García, Upgrade of an artificial neural network prediction method for electrical consumption forecasting using an hourly temperature curve model. Energy and Buildings. 2012. Under review.

[3] Moghaddam, M. Parsa; Abdollahi, A.; Rashidinejad, M., Flexible demand response programs modeling in competitive electricity markets. Applied Energy, 2011, Vol. 88(9), p. 3257-3269.

[4] Bompard, Ettore Napoli, Roberto; Wan, Bo, The effect of the programs for demand response incentives in competitive electricity markets. European Transaction on Electrical Power, 2009, Vol. 19(1), p. 127-139. 
[5] Moutinho, Victor; Vieira, Joel; Moreira, Antonio Carrizo, The crucial relationship among energy commodity prizes: Evidence from the Spanish electricity market. Energy Policy, Oct. 2011, Vol. 39(10), p. 5898-5908.

[6] Guiterman, Tim; Krarti, Moncef, Analysis of measurement and verification methods for energy retrofits applied to residential buildings. ASHRAE Transactions, July 2011, Vol. 117 (2), p. 382(13).

[7] Kumar, Satish, Measurement and Verification of Energy Savings. Energy Users News, Dec. 2000, Vol. 25(12), p. 24.

[8] Meyers, Steven Kromer, Steve, Measurement and verification strategies for energy savings certificates: meeting the challenges of an uncertain world. Energy Efficiency, 2008, Vol. 1(4), p. 313-321. 